\title{
The discovery of potential acetylcholinesterase inhibitors: A combination of pharmacophore modeling, virtual screening, and molecular docking studies
}

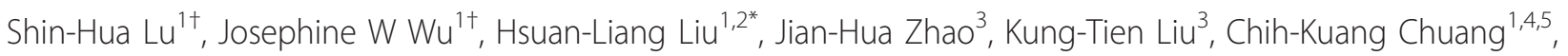
Hsin-Yi Lin', Wei-Bor Tsai ${ }^{6}$, Yih $\mathrm{Ho}^{7}$

\begin{abstract}
Background: Alzheimer's disease (AD) is the most common cause of dementia characterized by progressive cognitive impairment in the elderly people. The most dramatic abnormalities are those of the cholinergic system. Acetylcholinesterase (AChE) plays a key role in the regulation of the cholinergic system, and hence, inhibition of AChE has emerged as one of the most promising strategies for the treatment of AD.

Methods: In this study, we suggest a workflow for the identification and prioritization of potential compounds targeted against AChE. In order to elucidate the essential structural features for AChE, three-dimensional pharmacophore models were constructed using Discovery Studio 2.5.5 (DS 2.5.5) program based on a set of known AChE inhibitors.

Results: The best five-features pharmacophore model, which includes one hydrogen bond donor and four hydrophobic features, was generated from a training set of 62 compounds that yielded a correlation coefficient of $R=0.851$ and a high prediction of fit values for a set of 26 test molecules with a correlation of $R^{2}=0.830$. Our pharmacophore model also has a high Güner-Henry score and enrichment factor. Virtual screening performed on the $\mathrm{NCl}$ database obtained new inhibitors which have the potential to inhibit AChE and to protect neurons from $A \beta$ toxicity. The hit compounds were subsequently subjected to molecular docking and evaluated by consensus scoring function, which resulted in 9 compounds with high pharmacophore fit values and predicted biological activity scores. These compounds showed interactions with important residues at the active site.
\end{abstract}

Conclusions: The information gained from this study may assist in the discovery of potential AChE inhibitors that are highly selective for its dual binding sites.

\section{Background}

Acetylcholinesterase (AChE), one of the most essential enzymes in the family of serine hydrolases, catalyzes the hydrolysis of neurotransmitter acetylcholine, which plays a key role in memory and cognition [1-3]. While the physiological role of the AChE in neural transmission has been well known, it is still the focus of pharmaceutical research, targeting in treatments of myasthenia

\footnotetext{
* Correspondence: f10894@ntut.edu.tw

+ Contributed equally

'Graduate Institute of Biotechnology, National Taipei University of Technology, 1 Sec. 3 ZhongXiao E. Rd., Taipei, 10608, Taiwan Full list of author information is available at the end of the article
}

gravis, glaucoma, and Alzheimer's disease (AD). It has been elucidated that cholinergic deficiency is associated with AD [4]; therefore, one of the major therapeutic strategies is to inhibit the biological activity of AChE, and hence, to increase the acetylcholine level in the brain. Currently, most of the drugs used for the treatment of $\mathrm{AD}$ are $\mathrm{AChE}$ inhibitors, including the synthetic compounds tacrine, donepezil, and rivastigmine, which have all been proven to improve the situation of $\mathrm{AD}$ patients to some extent. So far, the four drugs that have been approved by the Food and Drug Administration (FDA) to treat $\mathrm{AD}$ in the US are tacrine, rivastigmine 
(E2020), donepezil, and galanthamine, which all have some success in slowing down neurodegeneration in $\mathrm{AD}$ patients.

In the past decade, it has been found that $\mathrm{AChE}$ is involved in pathogenesis of $\mathrm{AD}$ through a secondary noncholinergic function associated with its peripheral anionic site. Recent findings support the enzyme's role in mediating the processing and deposition of $A \beta$ peptide by colocalizing with $A \beta$ peptide deposits in the brain of $A D$ patients and promoting $A \beta$ fibrillogenesis through the formation of stable AChE- $\mathrm{A} \beta$ complexes. The formation of these complexes promotes $A \beta$ aggregation as an early event in the neurodegenerative cascade of $\mathrm{AD}[5,6]$ and results in cognitive impairment in doubly transgenic mice expressing human amyloid precursor protein (APP) and human AChE $[7,8]$. Based on these new findings, the recent design of novel classes of $\mathrm{AChE}$ inhibitors as therapeutic intervention for AD has been shifted toward blocking the peripheral site of $A C h E$, the $A \beta$ recognition zone within the enzyme [9], thereby affect the $A C h E$-induced $A \beta$ aggregation and thus, modulate the progression of $\mathrm{AD}$.

$\mathrm{X}$-ray structures of AChE co-crystallized with various ligands [10-14] provided insights into the essential structural elements and motifs central to its catalytic mechanism and mode of acetylcholine ( $\mathrm{ACh}$ ) processing. One of the striking structural features of the AChE revealed from the $\mathrm{X}$-ray analysis is the presence of a narrow, long, hydrophobic gorge which is approximately $20 \AA$ deep $[15,16]$. The enzyme has a catalytic triad consisting of Ser203, His447, and Glu334 [17] located in the active site of the narrow deep gorge, the lining of which contains mostly aromatic residues that form a narrow entrance to the catalytic Ser203 [16]. A peripheral anionic site (PAS) comprising another set of aromatic residues Tyr72, Tyr124, Trp286, Tyr341, and Asp74 [18] is located at the rim of the gorge and provides a binding site for allosteric modulators and inhibitors. The interaction between highly potent inhibitors, such as tacrine and donepezil, and the enzyme is characterized by cation- $\pi$ interactions between the protonated nitrogens and the conserved aromatic residues, tryptophan (Trp86) and phenylalanine (Phe337). Moreover, $\pi-\pi$ stacking between the aromatic moieties of the inhibitors and the aromatic amino acids mentioned above, as well as ion-ion-interactions between the protonated nitrogens of the inhibitors and the anionic aspartic acid (Asp72) all play crucial roles in ligand binding [15]. Most ligands, as observed from their crystal structures, are located at the bottom of the gorge that forms a wide hydrophobic pocket base, although larger ligands such as decamethonium [10] and donepezil [19] extend to the mouth of the gorge, the opening of the hydrophobic pocket.
The drug discovery process is both time-consuming and expensive [20] yet new drugs are required to satisfy the numerous unmet clinical needs in many disease indications. The number of potential target 3D structures is increasing in the Protein Data Bank (PDB) [19] and the number of drug/lead-like compounds is estimated to be at least $10^{24}$ [21]. Therefore, to deal with such a large amount of data and to facilitate the drug discovery process, in silico virtual screening and computer-aided drug design have become increasingly important [22]. Virtual screening provides an inexpensive and fast alternative to high-throughput screening for discovering new drugs. The binding of ligand to receptor is driven in part by shape complementarity and physicochemical interactions. One of the virtual screening approaches is to develop a pharmacophore query from an inhibitor, which describes the spatial arrangement of a group of essential structural features common to a set of compounds that are critical to interacting with the receptor. The pharmacophore approach is applied in drug design and takes in consideration that molecules are active at the receptor binding site because they possess both a number of chemical features that favor the target interaction site and are geometrically complementary to it. A good pharmacophore model collects important common features of molecules distributed in the 3D space and provides a rational hypothetical conformation of the primary chemical features responsible for activity; therefore, it has become an important method and has proven extremely successful not only in demonstrating structure-activity relationships, but also in the development of new drugs [23,24].

Providing that the experimentally determined highresolution 3D structure of the target is available, ligandbased drug design can be performed in association with molecular docking, a structure-based method, and underlying scoring functions to reproduce crystallographic ligand-binding modes. These methods can be combined to identify a number of new hit compounds with potent inhibitory activity and to understand the main interactions at the binding sites. It is believed that the concurrent use of molecular docking and consensus scoring functions could readily minimize false positive and false negative errors encountered by ligand-based (pharmacophore) virtual screening. In addition, the complementation of molecular docking and pharmacophore can produce reliable true positive and true negative results in the subsequent virtual screening procedure. The appropriate use of these methods in a drug discovery process should improve the ability to identify and optimize hits and confirm their potential to serve as scaffolds for producing new therapeutic agents.

In this study, we developed both qualitative and quantitative pharmacophore models based on AChE inhibitors 
collected from the same laboratory [25-33]. The pharmacophore features were used to identify potent AChE inhibitors as well as to clarify the quantitative structureactivity relationship for previously known AChE inhibitors. The best quantitative model was used as 3D search queries for screening the NCI databases to identify new inhibitors of $\mathrm{AChE}$ that can block both the catalytic and peripherical anionic sites. Blocking the daul-binding sites has the advantages of both preventing the degradation of acetylcholine in the brain and inhibiting the pro-aggregating effect of $\mathrm{AChE}$, thus, protect neurons from $A \beta$ toxicity. Once identified, the hit compounds were subsequently subjected to filtering by molecular docking to refine the retrieved hits. The virtual screening approach, in combination with pharmacophore modeling, molecular docking, and consensus scoring function can be used to identify and design novel AChE inhibitors with higher selectivity. The potential hit compounds obtained from this study can be further evaluated by in vitro and in vivo biological tests.

\section{Methods}

\section{Data preparation}

Pharmacophore modeling correlates activities with the spatial arrangement of various chemical features in a set of active analogues. The $88 \mathrm{AChE}$ inhibitors in this study were collected from nine publications reported by the same laboratory [25-33], which employed similar experimental conditions and procedures to obtain bioactivity data for the compounds. The in vitro bioactivities of the collected inhibitors were expressed as the concentration of the test compounds that inhibited the activity of AChE by $50 \%$ (IC50). These values are generally transformed into pIC50 (-log IC50) as an expression of drug potency. Additional files 1 and 2 (Tables S1 and S2) show the structures, IC50 and pIC50 values of the inhibitors considered for this study. Among these sets, 62 diverse compounds whose binding affinities (IC50 values) ranged from $0.00106 \mu \mathrm{M}$ to $80.5 \mu \mathrm{M}$ (over six orders of magnitude) were selected as the training set (Additional file 1: Table S1); while the remaining 26 molecules served as the test set (Additional file 2: Table S2). The training set molecules play an important role in determining the quality of the pharmacophore models generated; while the test set compounds serve to evaluate the predictive ability of the resultant pharmacophore. Both sets of molecules must have large range of activities to obtain critical information on the pharmacophoric requirements for $\mathrm{AChE}$ inhibition.

The two-dimensional (2D) chemical structures of these acetylcholinesterase inhibitors (AChEIs) were sketched using CS ChemDraw Ultra (Cambridge Soft Corp., Cambridge, MA) and saved as MDL-molfile format. Subsequently, they were imported into Discovery
Studio Version 2.5.5 (DS 2.5.5, Accelrys Inc., San Diego, CA) and converted into the corresponding standard three-dimensional (3D) structures. Molecular flexibility of compounds is modeled by making multiple conformers within a specific energy range. A maximum of 250 conformers for each compound were generated by the "Best quality" conformational search option based on the CHARMm force field [34], with an energy threshold of $20 \mathrm{kcal} / \mathrm{mol}$ from the lowest energy level. Default settings were kept for the other parameters.

\section{Pharmacophore model generation}

Two different methods were applied for the ligand based pharmacophore model: HipHop and HypoGen. HipHop is generated based on the common features present in the training set molecules. HypoGen [35], an algorithm that uses the activity values of the small compounds in the training set to generate the hypothesis, was applied in this study to build the 3D QSAR pharmacophore models using DS V2.5.5 software. An automated 3D QSAR pharmacophore was created by using the activity values of compounds in the training set that includes at least 16 molecules with bioactivities spanning at least over four orders of magnitude. The wide range of bioactivities in the training set allowed for the screening of large database. The DS Feature Mapping module computed all possible pharmacophore feature mappings for the selected chemical features of the training set molecules. A minimum of 0 to a maximum of 5 features including hydrogen-bond acceptor (HBA), hydrogenbond donor (HBD), hydrophobic (HBic), and ring aromatic (RingArom) features were selected in generating the quantitative pharmacophore model. A value of 3 was employed as the uncertainty value, which means that the biological activity of a particular inhibitor is assumed to be located somewhere in the range three times higher to three times lower of the true value of that inhibitor [35-38]. Ten pharmacophore models with significant statistical parameters were generated. The best model was selected on the basis of a highest correlation coefficient $(\mathrm{R})$, lowest total cost and root mean square deviation (rmsd) values (for more details on cost values, see Ref. [39]). From the pharmacophore models generated, the relationship between the structures of the training set compounds and their experimentally determined inhibitory activities against AChE was investigated.

\section{Validation of the pharamacophore model}

The pharmacophore models selected by correlation coefficient and cost analysis were then validated in three subsequent steps: Fischer's randomization test, test set prediction, and Güner-Henry $(\mathrm{GH})$ scoring method [40-42]. First, cross validation was performed by 
randomizing the data using the Fischer's randomization test. Then, a test set of 26 diverse compounds with AChE inhibitory activity was selected to validate the best pharmacophore model. The test set covers similar structural diversity as the training set in order to establish the broadness of the pharmacophore predictability. All queries were performed using the Ligand Pharmacophore Mapping protocol. The GH scoring method was used following test set validation to assess the quality of the pharmacophore models. The GH score has been successfully applied to quantify model selectivity precision of hits and the recall of actives from a 3,606 molecule dataset consisting of known actives and in-actives. Of these molecules, 66 structurally and pharmacologically diverse compounds are known inhibitors of AChE that were selected from four publications [43-46]. While the other 3,540 molecules were from the previously published directory of useful decoys (DUD) dataset [47]. The DUD database, which is available for public use, was generated based on the observation that physical characteristics of the decoy background can be used for the classification of different compounds. DUD was downloaded from http://dud.docking.org (accessed July 17, 2010).

The GH scoring method was applied to the previously mentioned 66 known inhibitors of AChE and the DUD dataset molecules to validate the pharmacophore models. The method consists of computing the following: the percent yield of actives in a database (\%Y, recall), the percent ratio of actives in the hit list (\% A, precision), the enrichment factor $E$, and the $\mathrm{GH}$ score. The $\mathrm{GH}$ score ranges from 0 to 1 , where a value of 1 signifies the ideal model.

The following is the proposed metrics for analyzing hit lists by a pharmacophore model-based database search [40-42]:

$$
\begin{gathered}
\% \mathrm{~A}=\frac{\mathrm{Ha}}{\mathrm{A}} \times 100 \\
\% \mathrm{Y}=\frac{\mathrm{Ha}}{\mathrm{Ht}} \times 100 \\
\mathrm{E}=\frac{\mathrm{Ha} / \mathrm{Ht}}{\mathrm{A} / \mathrm{D}} \\
\mathrm{GH}=\left(\frac{\mathrm{Ha}(3 \mathrm{~A}+\mathrm{Ht})}{4 \mathrm{HtA}}\right)\left(1-\frac{\mathrm{Ht}-\mathrm{Ha}}{\mathrm{D}-\mathrm{A}}\right)
\end{gathered}
$$

$\% \mathrm{~A}$ is the percentage of known active compounds retrieved from the database (precision); $\mathrm{Ha}$, the number of actives in the hit list (true positives); A, the number of active compounds in the database; \%Y, the percentage of known actives in the hit list (recall); Ht, the number of hits retrieved; $\mathrm{D}$, the number of compounds in the database; E, the enrichment of the concentration of actives by the model relative to random screening without any pharmacophoric approach and GH is the GünerHenry score.

\section{Virtual screening}

Virtual screening, an in silico tool for drug discovery, has been widely used for lead identification in drug discovery programs. Virtual screening methods are generally divided into ligand-based virtual screening and structure-based virtual screening. Pharmacophore-based database searching is considered a type of ligand-based virtual screening, which can be efficiently used to find novel, potential leads for further development from a virtual database. A well-validated pharmacophore model includes the chemical functionalities responsible for bioactivities of potential drugs, therefore, it can be used to perform a database search by serving as a $3 \mathrm{D}$ query. The best pharmacophore Hypo1 was used as a 3D structural query for retrieving potent molecules from the NCI chemical database. For each molecule in the database, the fast conformer generation method produced 250 conformers with a maximum energy tolerance of $20 \mathrm{kcal} / \mathrm{mol}$ above that of the most stable conformation.

The compounds were first filtered by Lipinski's "Rule of five" that sets the criteria for drug-like properties. Drug likeness is a property that is most often used to characterize novel lead compounds [48] by screening of structural libraries. According to this rule, poor absorption is expected if $\mathrm{MW}>500, \log \mathrm{P}>5$, hydrogen bond donors $>5$, and hydrogen bond acceptors $>10$ [49]. Secondly, a molecule that satisfied all the features of the pharmacophore model used as the 3D query in database searching was retained as a hit. Two database searching options such as Fast/Flexible and Best/Flexible search are available in DS V2.5.5. Of these two, the "Best/Flexible search" yielded better results during database screening, therefore, we performed all database searching experiments using the "Best/Flexible search" option. Setting the "Maximum Omitted Features" option to zero, the best pharmacophore model was used to screen the databases for those compounds that fit all five features of the pharmacophore Hypo1. The calculations of fit values were based on how well the chemical substructures match the location constraints of the pharmacophoric features and their distance deviation from the feature centers. High fit values indicate good matches. The maximum fit value was set based on the fit value of the original ligands used to create the pharmacophore models. Those hit compounds that passed all of the screening tests were taken for further molecular docking study. 


\section{Molecular docking}

The DOCK protocols used in this study were the procedures described in our laboratory, and the methodology for their preparation has been previously studied (unpublished results). Crystal structure of AChE (PDB code: 1B41) [50], downloaded from the protein databank (PDB) [19], was used for the study. The solvent molecules were removed and hydrogen atoms were added to the protein using DS V2.5.5. Structure-based docking of 88 minimized AChE inhibitors and hits/leads from virtual screening to the active site of AChE was carried out using the LibDock program [51], which is an extension of the software DS V2.5.5. The active site was defined as the region of $\mathrm{AChE}$ that comes within $12 \AA$ from the geometric centroid of the ligand. Default settings for small molecule-protein docking were used throughout the simulations. Top 50 poses were collected for each molecule with the best docked score value associated with a favorable binding conformation compared to the co-crystallized inhibitor being considered as having biological activity.

\section{Results}

\section{Construction of pharmacophore model}

Before the start of pharmacophore modeling, we collected a total of $88 \mathrm{AChE}$ inhibitors from different literature resources. Of these compounds, 62 were carefully chosen to form a training set based on wide coverage of activity range and structural diversity. Structures and biological activities of the training set compounds are shown in Additional file 1: Table S1. The remaining compounds were included in the test set (see Additional file 2: Table S2). The top ten hypotheses were composed of HBA, HBD, HBic, and RingArom features. The values of the ten hypotheses such as pharmacophore features, root-mean-square deviations (rmsd), correlation ( $r$ ), cost values, and Fischer confidence levels showed statistical significance (Table 1).

The best hypothesis Hypo1, as shown in Figure 1, is characterized by the lowest total cost value (289.972), the highest cost difference (142.57), the lowest RMSD (1.411), and the best correlation coefficient $(R=0.851)$. The fixed cost and null cost are 228.233 and 432.542 bits, respectively. The total cost is low and close to the fixed cost, as well as being less and differs greatly from the null cost. All of these evidence indicate that the model, accounting for all five pharmacophore features: one hydrogen bond donor (HBD) and four hydrophobic (HBic), has good predictive ability. Figures $1 \mathrm{~A}$ and $1 \mathrm{~B}$ show the 3D spatial arrangement and distance constraints of all HypoGen pharmacophore features in Hypo1. The features of Hypo1 (HBD and HBic) were mapped onto the most active compound of the training set (compound 7) shown in Figure $1 \mathrm{C}$. One of the low active compound in the training set (compound 44) was mapped partially by the features of Hypo1 (Figure 1D). Clearly, all features in the hypothesis are mapped very well with the corresponding chemical functional groups on compound 7, while three features (i.e. one hydrogenbond donor and two hydrophobic features) are not mapped to any functional group on compound 44. The results of our pharmacophore study appear to validate the Hypo1 model to some extent.

\section{Model validation}

The pharmacophore model constructed in this study was primarily validated to check for the best model that can identify the active compounds in a virtual screening process. The three steps of validation include Fischer's randomization test method, correlation of the experimental activity and the estimated fit values of the test set, and Güner-Henry (GH) scoring method.

All hypotheses were then evaluated by cross-validation using Fischer's randomization method. Validation was done by generating 19 random spreadsheets (95\% confidence level) for the training set molecules and randomly reassigning activity values to each compound. The same method was used for each hypothesis to generate the random spreadsheets. The cross-validated experiment confirmed that the hypotheses have 95\% significance and the results are shown in Table 1 . The high statistical significance may be attributed to the significant difference between the activities of the training set molecules.

The pharmacophore model should estimate the predicted fit values of the training set molecules and accurately predict the fit values of the test set molecules. First, all ten hypotheses were evaluated using a test set of 26 known AChE inhibitors. Fit values were calculated using all ten hypotheses and correlated with experimental activities. The best hypothesis, Hypo1, showed a correlation coefficient $\left(\mathrm{R}^{2}=0.830\right)$. The correlation between the experimentally observed and estimated fit values for the training set and the test set molecules is plotted in Figure 2.

Another statistical test method used for validation includes calculation of false positives, false negatives, enrichment, and goodness of hit to determine the robustness of the generated hypotheses. Not only should the pharmacophore model generated predict the activity of the training set compounds, but it should also be capable of predicting the activities of other compounds as active or inactive. Hypo1 was used to search the known AChE inhibitors through database mining by using the BEST flexible searching technique. The results were analyzed using the hit list $(\mathrm{Ht})$, number of active percent of yields $(\% \mathrm{Y})$, percent ratio of actives in the hit list $(\% \mathrm{~A})$, enrichment factor $(\mathrm{E})$, false negatives, false 
Table 1 The performance of 10 pharmacophoric hypotheses generated by HypoGen for AChE inhibitors

\begin{tabular}{|c|c|c|c|c|c|c|c|}
\hline \multirow[t]{2}{*}{ Hypotheses $^{a}$} & \multirow{2}{*}{$\begin{array}{l}\text { Pharmacophoric features in generated } \\
\text { hypotheses }\end{array}$} & \multirow{2}{*}{$\begin{array}{c}\text { RMS } \\
\text { deviation }\end{array}$} & \multicolumn{4}{|c|}{ Cost Values } & \multirow{2}{*}{$\begin{array}{l}\text { Residual } \\
\text { cost }^{d}\end{array}$} \\
\hline & & & Training set (R) & Error & Weight & Total $^{c}$ & \\
\hline 1 & $\mathrm{HBD}, 4 \times \mathrm{HBiC}$ & 1.411 & 0.851 & 270.24 & 1.170 & 289.972 & 142.57 \\
\hline 2 & $\mathrm{HBD}, 4 \times \mathrm{HBiC}$ & 1.416 & 0.850 & 270.73 & 1.338 & 290.628 & 141.91 \\
\hline 3 & $\mathrm{HBD}, 4 \times \mathrm{HBiC}$ & 1.419 & 0.849 & 270.97 & 1.144 & 290.681 & 141.86 \\
\hline 4 & $\mathrm{HBD}, 4 \times \mathrm{HBiC}$ & 1.445 & 0.843 & 273.31 & 1.419 & 293.293 & 139.25 \\
\hline 5 & HBD, 3xHBic, RingArom & 1.469 & 0.836 & 275.44 & 1.243 & 295.242 & 137.30 \\
\hline 6 & $\mathrm{HBD}, 4 \times \mathrm{HBic}$ & 1.474 & 0.834 & 275.93 & 1.145 & 295.636 & 136.91 \\
\hline 7 & $\mathrm{HBD}, 4 \times \mathrm{HBiC}$ & 1.484 & 0.834 & 276.83 & 1.219 & 296.614 & 135.93 \\
\hline 8 & $\mathrm{HBD}, 4 \times \mathrm{HBic}$ & 1.510 & 0.827 & 279.20 & 1.163 & 298.925 & 133.62 \\
\hline 9 & $\mathrm{HBD}, 4 \times \mathrm{HBiC}$ & 1.514 & 0.826 & 279.61 & 1.262 & 299.432 & 133.11 \\
\hline 10 & $\mathrm{HBD}, 4 \times \mathrm{HBiC}$ & 1.520 & 0.825 & 280.21 & 1.127 & 299.899 & 132.64 \\
\hline
\end{tabular}

${ }^{a}$ Fischer randomization set at $95 \%$ confidence level was performed on all pharmacophore models.

${ }^{\mathrm{b}}$ Correlation coefficient (R) between the experimental activity and the estimated fit values of the training compounds.

${ }^{c}$ Total costs $=$ error cost + weight cost + configuration cost, where configuration cost $=18.564$.

${ }^{d}$ Residual cost $=$ null cost - total cost, where null cost $=432.542$.

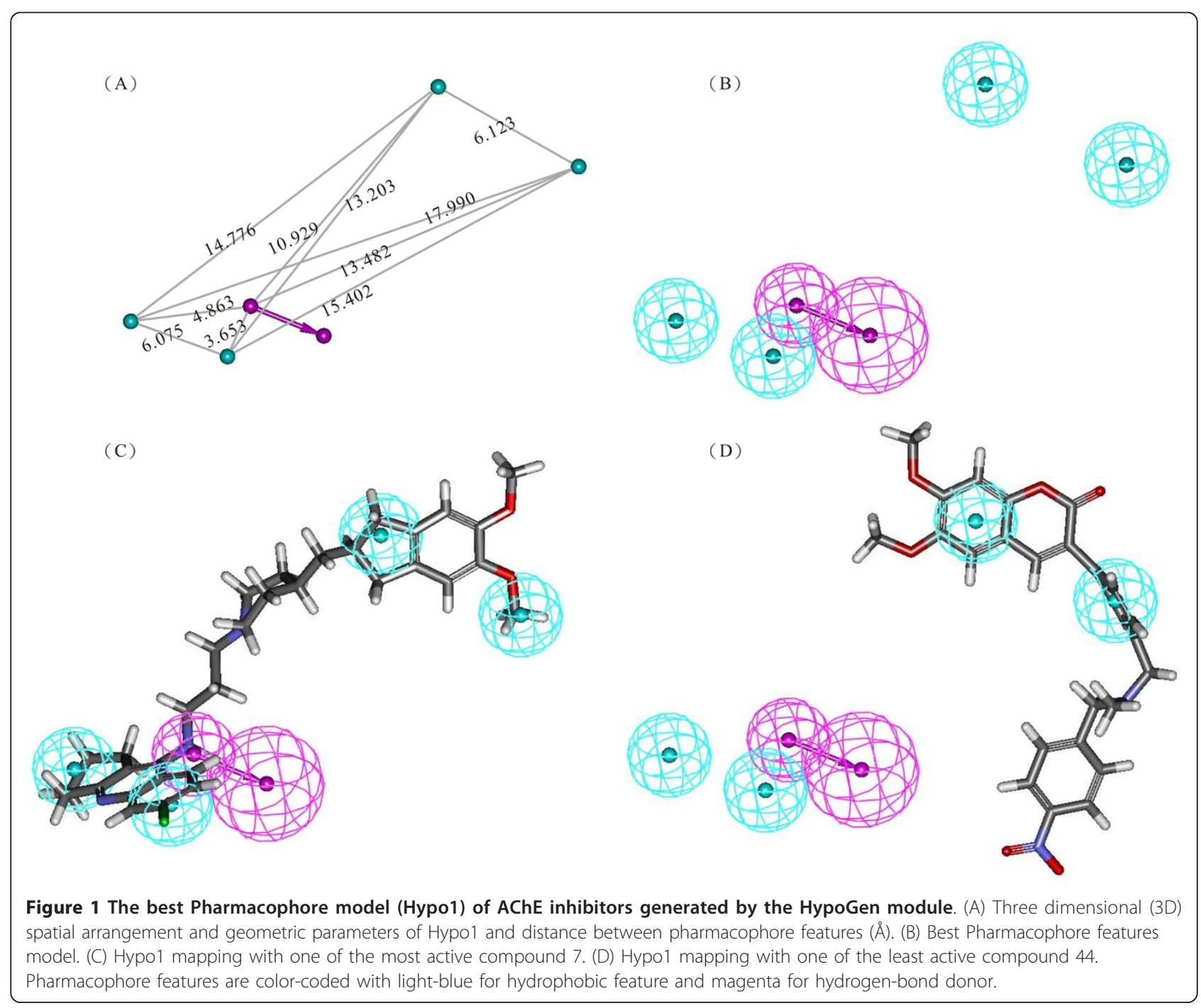



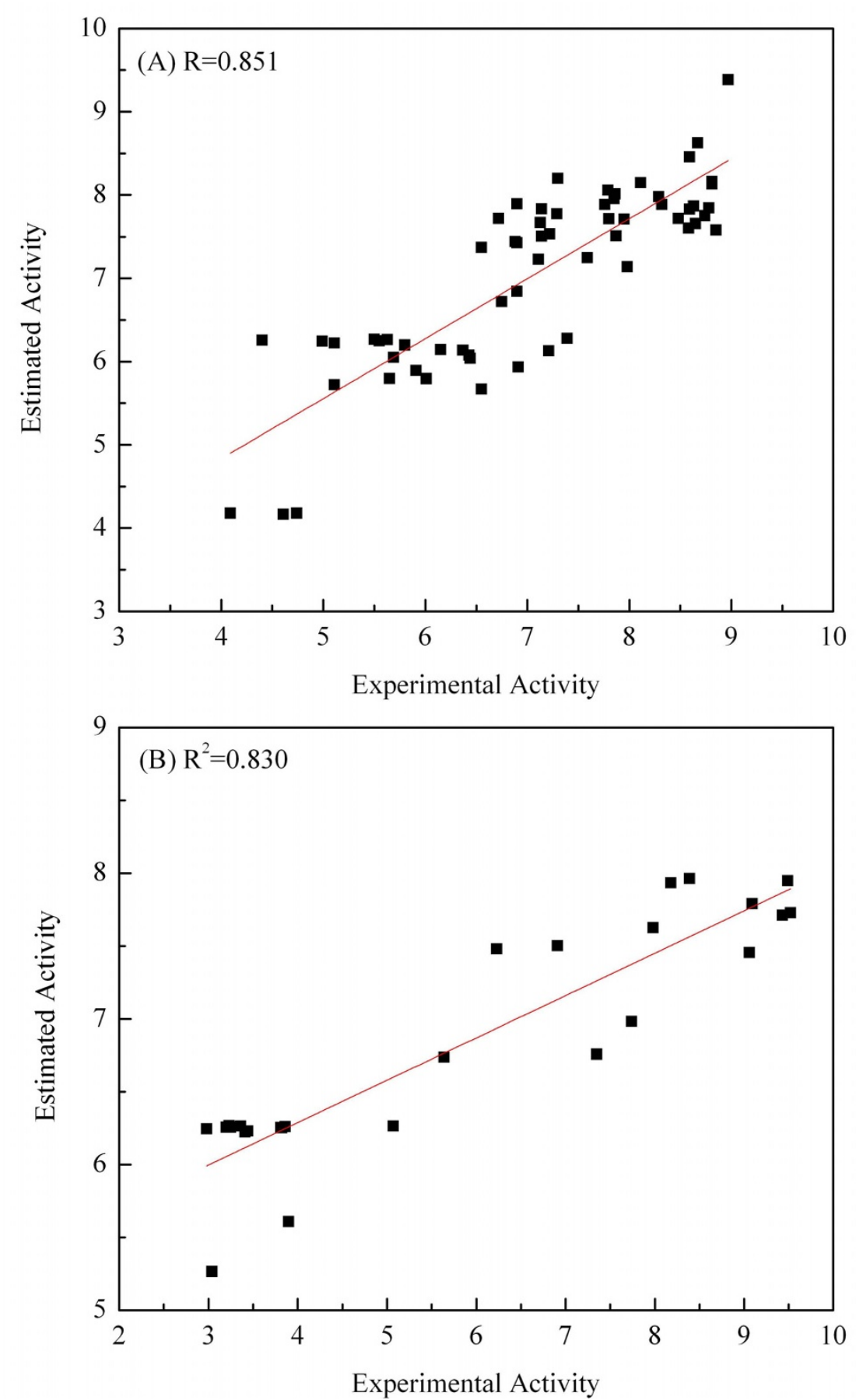

Figure 2 Plot of the correlation coefficient between experimental activity and estimated fit values by Hypo 1 . (A) The training set of 62 compounds $(R=0.851)$ and $(B)$ the test set of 26 compounds $\left(R^{2}=0.830\right)$.

positives, and goodness of hit score (GH scoring method) (Table 2). Hypo1 succeeded in retrieving 70\% of the active compounds, 22 inactive compounds (false positives), and predicted 13 active compounds as inactive (false negatives). An enrichment factor of 38.61 and a GH score of 0.73 indicated the quality of the model and high efficiency of the screening test. Overall, a strong correlation was observed between the Hypol predicted activity and the experimental AChE inhibitory activity (IC50) of the training and test set compounds (Figure 2). Fischer's randomization method also confirmed that the hypothesis has $95 \%$ significance, and the 
Table 2 Pharmacophore model evaluation based on the Güner-Henry scoring method

\begin{tabular}{lll}
\hline Serial No. & Parameter & AChE \\
\hline 1 & Total molecules in database (D) & 3606 \\
2 & Total Number of active in database (A) & 66 \\
3 & Total Hits $(\mathrm{Ht})$ & 75 \\
4 & Active Hits $(\mathrm{Ha})$ & 53 \\
5 & \% Yield of actives $[(\mathrm{Ha} / \mathrm{Ht}) \times 100]$ & 70.67 \\
6 & \% Ratio of actives $[(\mathrm{Ha} / \mathrm{A}) \times 100]$ & 80.30 \\
7 & Enrichment factor $(\mathrm{E})[(\mathrm{Ha} \times \mathrm{D}) /(\mathrm{Ht} \times \mathrm{A})]$ & 38.61 \\
8 & False negatives $[\mathrm{A}-\mathrm{Ha}]$ & 13 \\
9 & False positives $[\mathrm{Ht}-\mathrm{Ha}]$ & 22 \\
10 & Goodness of hit score & 0.73 \\
\hline
\end{tabular}

${ }^{a}[(\mathrm{Ha} / 4 \mathrm{HtA})(3 \mathrm{AtHt})) \times(1-((\mathrm{Ht}-\mathrm{Ha}) /(\mathrm{D}-\mathrm{A}))] ; \mathrm{GH}$ Score of $0.7-0.8$ indicates a very good model.

GH scoring method showed that the model can accurately screen for compounds with activity. These three validation procedures provided strong support for Hypo1 as the best pharmacophore model.

\section{Database screening}

One proficient approach to drug discovery is virtual screening of molecule libraries [52]. For conducting virtual screening, we used NCI database containing 260,071 compounds (accessed July 17, 2010). These compounds were first screened for drug like properties using Lipinski rule of 5 as filter [49]. The remaining 190,239 compounds that passed the screening were overlaid with the best 3D pharmacophore model (Hypo1) by using the 'Best Fit' selection. The top 252 hits with the highest fit values were subsequently analyzed for binding patterns using docking methods. The flowchart in Figure 3 is a schematic representation of the sequential virtual screening process with the number of hits reduced for each screening step.

\section{Molecular docking studies of AChE}

Docking simulation of AChE (PDB Code: 1B41) [50] and ligands was performed using the LibDock program. The binding modes for the 252 compounds identified by virtual screening were ranked according to the information obtained by different scoring constraints. The 154 highest scoring compounds were selected from a total of 252 compounds for further evaluation. After visual inspection, the most favorable compounds with the best binding modes (exact matching of $\pi-\pi$ overlap with residue W86 or $\pi-\pi$ overlap with residue W286) and structural diversity were selected. Based on the knowledge of the existing $\mathrm{AChE}$ inhibitors and the active site requirements, we selected 9 compounds from the 252 highest scoring structures for subsequent bioactivity prediction and consensus scoring function assay. Information on the molecular docking experiments and the consensus scoring function were taken from a previous study. The 9 hits with the highest binding affinities were ultimately selected after careful observations, analyses and comparisons. The structures of these best hits from the final screening are reported in Figure 4. The highest pose scores extracted from the eleven default scoring methods and the predicted pIC50 values calculated by the consensus scoring function developed in this study for all of the 9 best hits are summarized in Table 3. Among the hits found were some novel structures. The diversity of the hits demonstrated that the pharmacophore model was able to retrieve hits with similar features to the existing AChE inhibitors as well as novel scaffolds.

\section{Discussion}

In this work, we first generated a qualitative pharmacophore model to effectively map the critical chemical features for AChE inhibitors. The resulting binding hypotheses were automatically ranked based on their "total cost" values, which is the sum of the three costs: error cost, weight cost and configuration cost. As the root mean square difference between the estimated and measured biological activities of the training set molecules increases, so does the error cost. Error cost provides the highest contribution to the total cost [35-38]. HypoGen also calculates the cost of the null hypothesis, with the assumption that there is no relationship between the estimated and measured biological activities. The residual cost (Table 1) is the difference between the cost of null hypothesis and the total cost. The larger the difference between the cost of the null hypothesis and total cost, the greater the likelihood that the correlation between the fit values and actual activities is not a random occurrence [35-38]. The 62 training set molecules were then mapped onto Hypo1 resulting in a correlation coefficient of 0.851 , which indicates a good correlation between the actual activities and estimated fit values (Figure 3).

The best pharmacophore model, Hypo1, consists of five features: one hydrogen bond donor and four hydrophobic features. The best quantitative pharmacophore model was further validated by Fischer's randomization test, test set prediction, and Güner-Henry (GH) scoring method. Results of Fischer's randomization test confirmed that the generated hypotheses from the training set are reasonable and that the Hypo1 pharmacophore model has been correctly established. The results obtained by the test set method show good correlation between the experimental activity and the estimated fit values (correlation coefficient of $R^{2}=0.830$ ) indicating that the pharmacophore model predicted molecular properties well. The results of GH scoring method show that the model is able to identify the active AChE compounds from the database. 


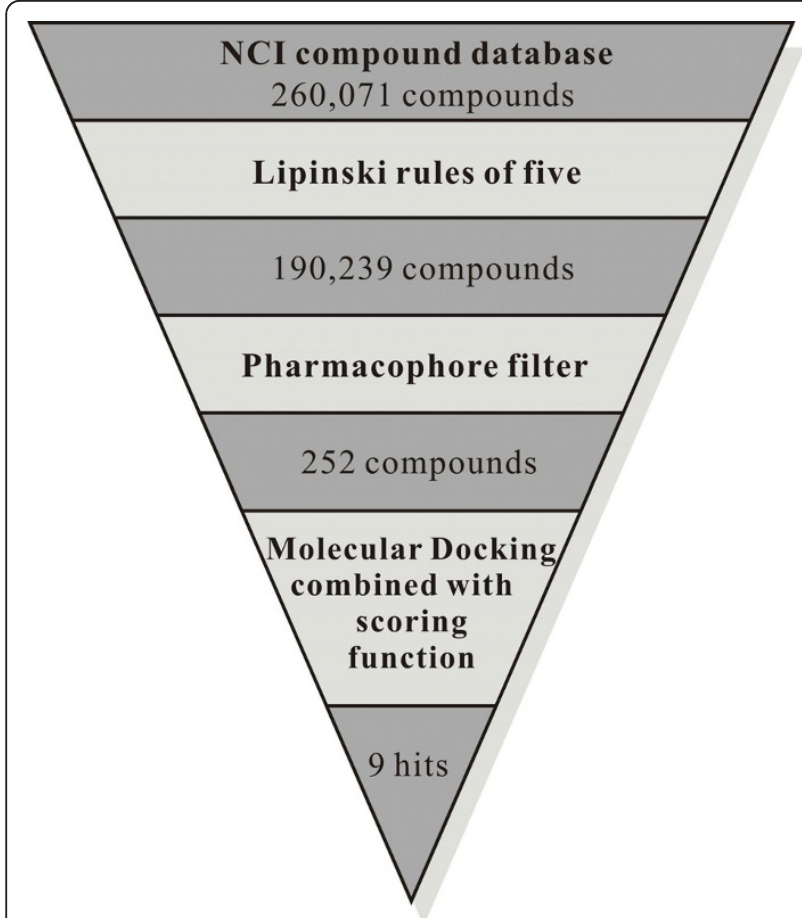

Figure 3 Schematic representation of virtual screening protocol implemented in the identification of $\mathrm{AChE}$ inhibitors.

Combining the best pharmacophore model, docking, and finally consensus scoring function activity prediction, we were able to perform virtual screening on a dataset of compounds to identify potential AChE inhibitors and to examine important interactions responsible for binding to AChE. The interactions of the best two compounds (NSC659829 and NSC35839) with the active site of huAChE protein are shown in Figure 5. Figure 6 maps out the interactions between the catalytic gorge of huAChE and the corresponding AChEIs presented in Figure 5 . The structure activity relationships of the best hit, NSC659829, against huAChE observed via docking interactions showed that the oxygen and nitrogen functionalities have strong hydrogen bond interactions with S203, G122 and Y124 amino acids present in the active site of huAChE and thus these groups are essential for activity. In the active site, the benzyl rings form a $\pi-\pi$ interaction with the indole ring of W86; while in the peripheral site (PS), the benzyl ring forms another $\pi-\pi$ interaction with the indole ring of W286.

Docking studies of the NSC35839 compound with huAChE revealed that the oxygen and nitrogen functionalities are making hydrogen interactions with the active site containing Y72, Y124, Y203 and Y337 amino acids. In the PAS, the benzyl ring forms another $\pi-\pi$ interaction with the indole ring of W286. Despite the lack of $\pi-\pi$ interaction with W86, other interactions were found to play important roles. Hydrogen bonds might be one reason for the enhanced activity of nitro substituted compounds. The proposed interactions of these compounds with W286 in huAChE suggest a possibility to interfere with amyloid fibrillogenesis in addition to inhibiting the catalytic function of the enzyme. The interactions found after docking include $\pi-\pi$ stacking contacts with residues in the anionic substrate binding site (Trp86, Phe331, and Tyr334) and the PAS (Trp286). Hydrogen bonding to amino acids is also found at the bottom of the gorge.

The combination of these interactions in other inhibitors (e.g., donepezil, galanthamine) is already found in the AChE crystal complex structure and therefore the docking results also show similarities that are meaningful for the test compounds. In addition, although all compounds are able to bind the active side of the gorge, not all of them are able to interact with all the important residues previously identified at the binding sites. Ligand size may be one reason for some of the activities being low. McCammon and coworkers have previously mentioned this problem with their molecular dynamics studies [53].

In conclusion, the previously mentioned $\pi-\pi$ interactions, hydrogen bonds, and strong hydrophobic interactions formed between the inhibitors and the nearby huAChE side chains serve dual roles: 1) to inhibit the catalytic activity of AChE by competing with Ach binding site and 2) to prevent amyloid fibrillogenesis by blocking the $A \beta$ recognition zone at the peripheral site. In light of the pharmacophore model developed in this study and the knowledge gained from the observations of the interactions between huAChE and potential inhibitors, it can be seen that the combination of pharmacophore, molecular docking, and virtual screening efforts is successful for discovering more effective inhibitory compounds that can have a great impact for future experimental studies in diseases associated AChE inhibition.

\section{Conclusions}

The work presented in this study shows that a set of compounds along with their activities ranging over several orders can be used to generate a good pharmacophore model, which in turn can be utilized to successfully predict the activity of a wide variety of chemical scaffolds. This model can then be used as a 3D query in database searches to determine compounds with various structures that can be effective as potent inhibitors and to assess how well newly designed compounds map onto the pharmacophore prior to undertaking any further research including synthesis.

Biological evaluation and optimization in designing or identifying compounds as potential inhibitors of AChE 


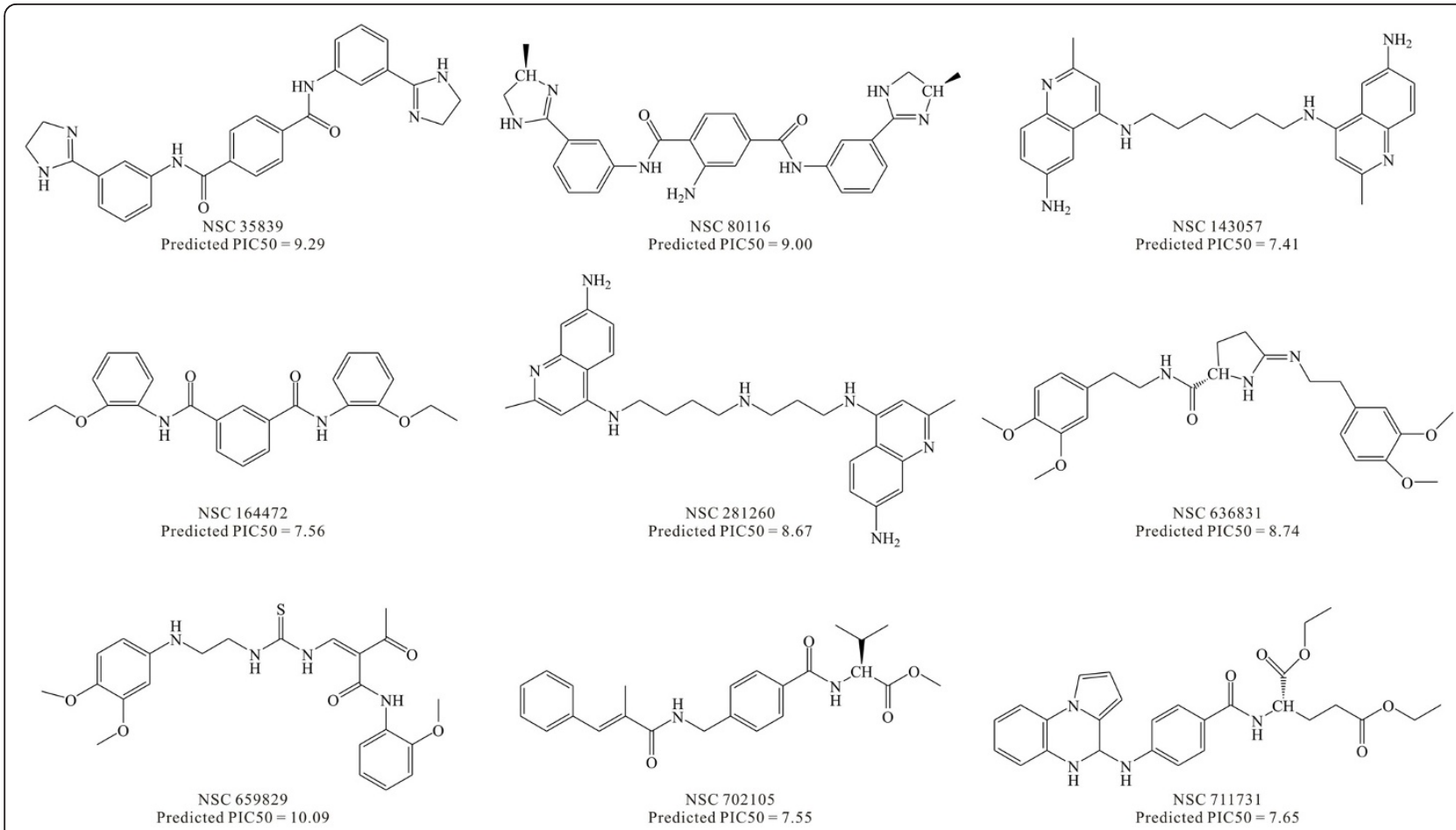

Figure 4 Lead molecules retrieved from the $\mathrm{NCl}$ database as potent AChE inhibitors. The predicted IC50 values are based on the consensus scoring function.

were made possible by the our pharmacophore study that showed the best model of AChE inhibitors were made up of one hydrogen bond donor and four hydrophobic features. The most active molecule in the training set fits the pharmacophore model perfectly with the highest scores. The pharmacophore model was further used to screen potential compounds from the NCI database followed by virtual screening that produced some number of false positives and false negatives. Then we used molecular docking and consensus scoring methods, as added tools for virtual screening to minimize these errors. Through our docking study, the important interactions between the potent inhibitors and the active site residues were determined. Using a combination of pharmacophore modeling, virtual screening, and molecular docking, we successfully identified putative novel $\mathrm{AChE}$ inhibitors, which can be further evaluated by in vitro and in vivo biological tests.

\section{Author details}

Both SL and JW are graduate students in the Graduate Institute of Biotechnology of National Taipei University of Technology under HLL's instruction. HLL is a distinguished professor in the Graduate Institute of

Table 3 The highest pose scores for the most potent AChE inhibitors from the $\mathrm{NCl}$ database

\begin{tabular}{llllllllllllll}
\hline Name & & (-PLP1) & (-PLP2) & (-PMF) & (-PMF04) & Jain & LibDockScore & LigScore1 & LigScore2 & Ludi1 & Ludi2 & Ludi3 & PIC50 \\
\hline NSC & 35839 & 128.01 & 127.24 & 259.45 & 188.38 & 5.78 & 152.48 & 3.65 & 4.53 & 808 & 624 & 1,333 & 9.29 \\
NSC & 80116 & 131.87 & 134.17 & 270.73 & 193.51 & 5.78 & 154.91 & 2.63 & 3.75 & 782 & 623 & 1,374 & 9.00 \\
NSC & 143057 & 141.36 & 138.40 & 215.12 & 163.47 & 6.01 & 162.90 & 3.79 & 4.49 & 691 & 572 & 1,069 & 7.41 \\
NSC & 164472 & 114.29 & 125.19 & 202.62 & 142.87 & 4.11 & 129.24 & 4.20 & 5.67 & 761 & 633 & 996 & 7.56 \\
NSC & 281260 & 134.57 & 139.96 & 207.72 & 149.91 & 8.11 & 169.53 & 0.68 & -0.58 & 674 & 588 & 1,138 & 8.67 \\
NSC & 636831 & 128.45 & 125.65 & 231.45 & 166.82 & 5.73 & 161.51 & 0.75 & 0.13 & 693 & 592 & 984 & 8.74 \\
NSC & 659829 & 139.17 & 142.52 & 207.47 & 158.28 & 2.75 & 143.76 & 4.30 & 3.91 & 826 & 640 & 734 & 10.09 \\
NSC & 702105 & 115.87 & 114.15 & 222.21 & 142.65 & 4.30 & 142.34 & 2.38 & 2.43 & 690 & 549 & 808 & 7.55 \\
NSC & 711731 & 131.92 & 132.18 & 189.08 & 130.37 & 8.01 & 151.50 & 1.32 & -0.82 & 619 & 500 & 840 & 7.65 \\
\hline
\end{tabular}

The pose scores are extracted from the eleven default scoring methods with the predicted plC50 values calculated from the consensus scoring function developed in this study. 


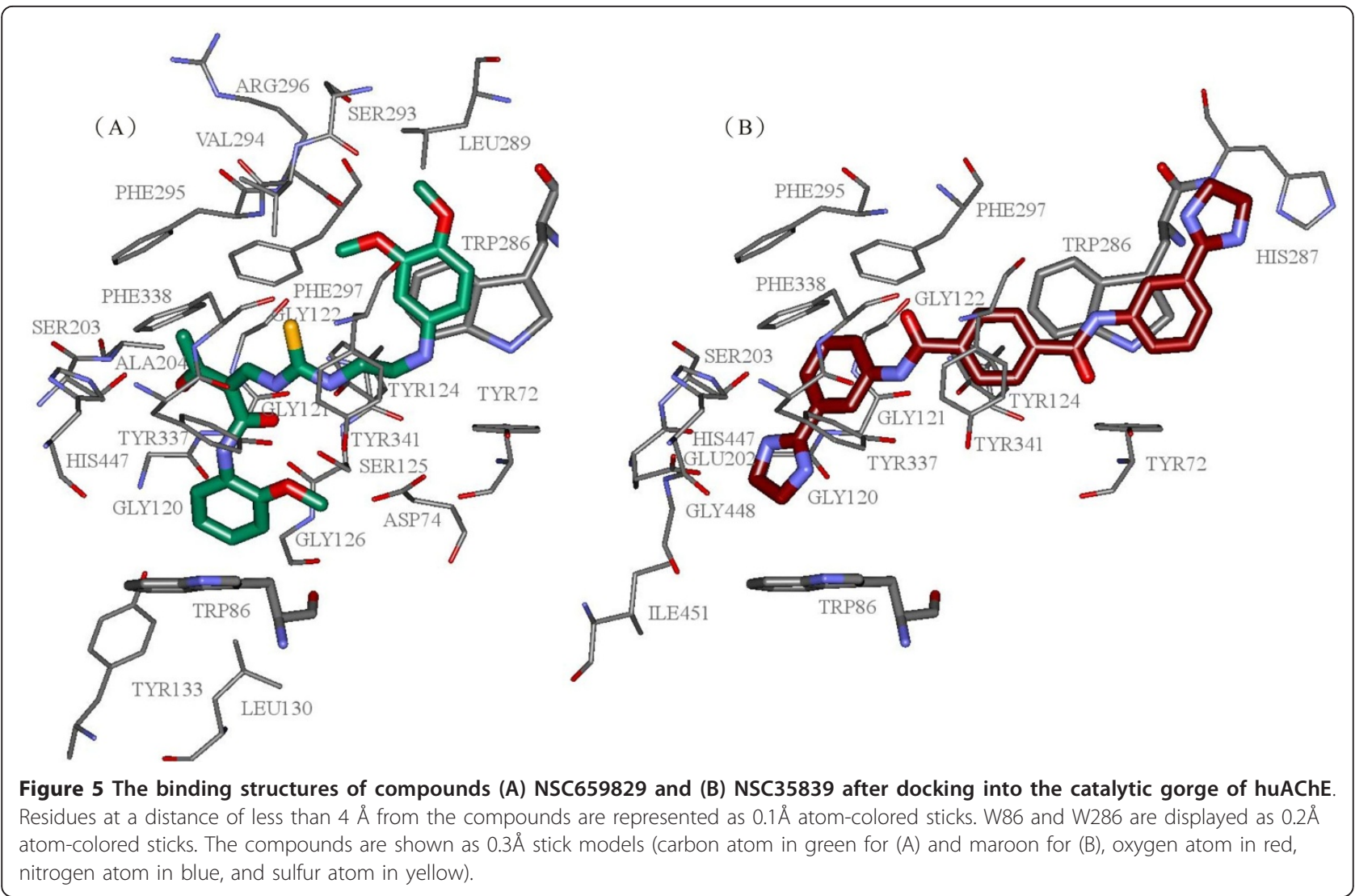

Biotechnology of National Taipei University of Technology. JZ is a postdoc fellow in the Chemical Analysis Division of Institute of Nuclear Energy Research under KL's instruction. KL works as a research fellow in the Chemical Analysis Division of Institute of Nuclear
Energy Research. CC is a research fellow in the Department of Medical Research of Mackay Memorial Hospital. HYL, WT, and YH are professors from National Taiwan University, National Taipei University of Technology, and Taipei Medical University, respectively.

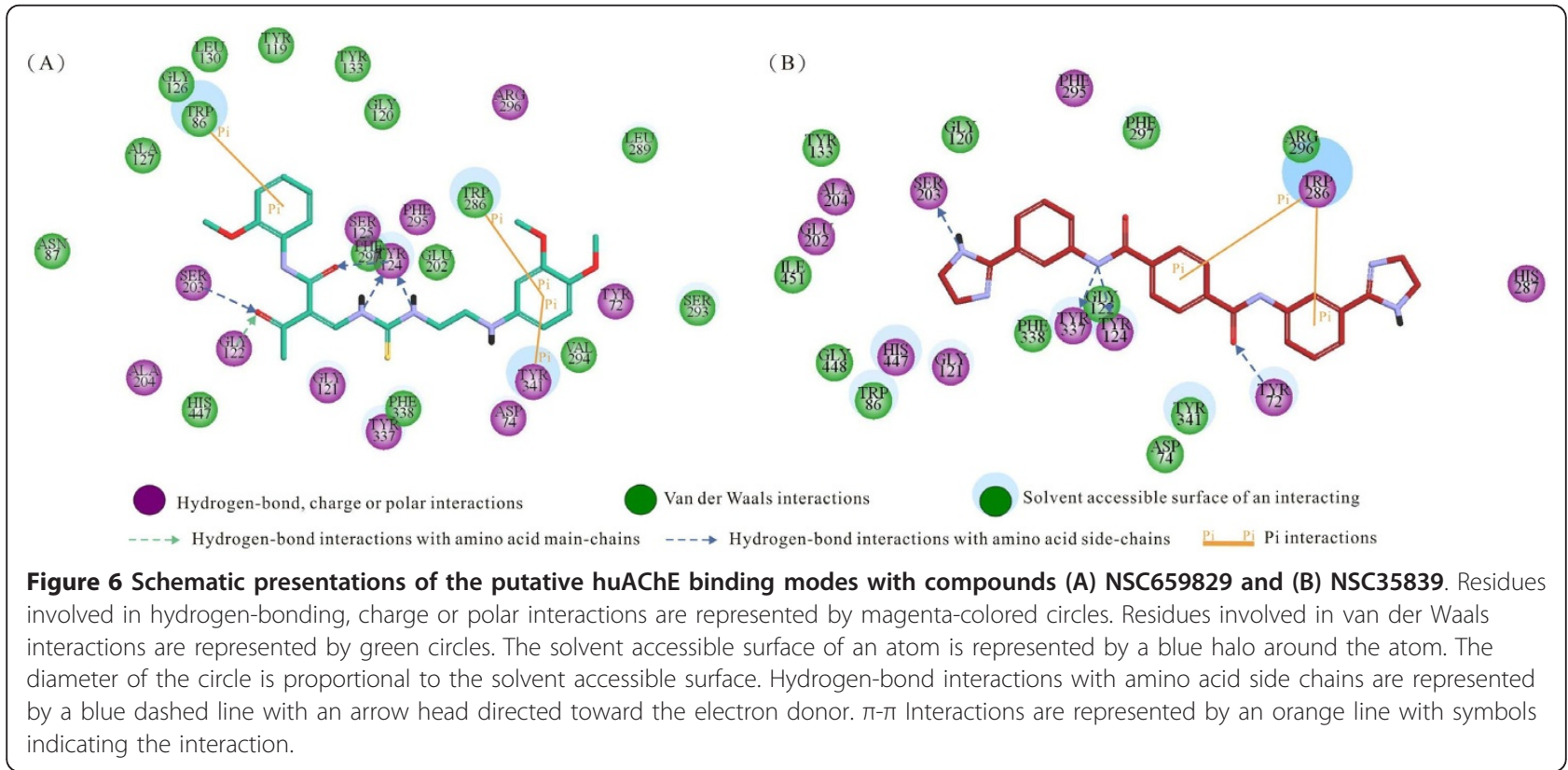




\section{Additional material}

Additional file 1: Table S1: The structures of AChE inhibitors utilized in the modeling. Training set of AChE inhibitors considered for this study, including chemical structures, experimental IC50 and pIC50 values.

Additional file 2: Table S2: The structures of AChE inhibitors utilized in the modeling. Test set of AChE inhibitors considered for this study, including chemical structures, experimental IC50 and plC50 values.

\section{Acknowledgements}

The authors gratefully acknowledge the financial supports from the National Science Council of Taiwan (Project numbers: 99-2221-E-027-022-MY3, 992221-E-027-037-MY2, NSC-96-2221-E-027-045-MY3, and 99-2622-E-027-003CC3), the Institute of Nuclear Energy Research of Taiwan (Project number: 992001INER072), and National Taipei University of Technology and Taipei Medical University (Project number: NTUT-TMU-98-02).

\section{Author details}

${ }^{1}$ Graduate Institute of Biotechnology, National Taipei University of Technology, 1 Sec. 3 ZhongXiao E. Rd., Taipei, 10608, Taiwan. ²Department of Chemical Engineering and Biotechnology, National Taipei University of Technology, 1 Sec. 3 ZhongXiao E. Rd., Taipei, 10608, Taiwan. ${ }^{3}$ Chemical Analysis Division, Institute of Nuclear Energy Research, 1000, Wunhua Rd., Longtan Township, Taoyuan County, 32546, Taiwan. ${ }^{4}$ Division of Genetics and Metabolism, Department of Medical Research, Mackay Memorial Hospital, 92, Sec. 2, Chung-Shan N. Rd., Taipei, 10449, Taiwan. ${ }^{5}$ College of Medicine, Fu-Jen Catholic University, 510 Chung Cheng Rd, Hsinchuang, Taipei County, 24205, Taiwan. ${ }^{6}$ Department of Chemical Engineering, National Taiwan University, 1 Sec. 4 Roosevelt Rd., Taipei, 106, Taiwan. ${ }^{7}$ School of Pharmacy, Taipei Medical University, 250 Wu-Hsing St., Taipei, 110, Taiwan.

\section{Authors' contributions}

SL carried out the entire simulation work presented in this study. JW helped to compose the manuscript. HLL is the corresponding author for this article and made substantial contributions to conception and design of the experiments. JZ participated in the design of the study and performed the statistical analysis. $\mathrm{KL}, \mathrm{CC}, \mathrm{HYL}, \mathrm{WT}$, and $\mathrm{YH}$ provide valuable discussion to this work and helped to draft the manuscript. All authors have read and approved the final manuscript.

\section{Competing interests}

The authors declare that they have no competing interests.

Received: 15 November 2010 Accepted: 21 January 2011 Published: 21 January 2011

\section{References}

1. Munoz-Muriedas J, Lopez JM, Orozco M, Luque FJ: Molecular modelling approaches to the design of acetylcholinesterase inhibitors: new challenges for the treatment of Alzheimer's disease. Curr Pharm Design 2004, 10:3131-3140.

2. Van Belle D, De Maria L, lurcu G, Wodak SJ: Pathways of ligand clearance in acetylcholinesterase by multiple copy sampling. J Mol Biol 2000, 298:705-726.

3. Xu Y, Colletier JP, Jiang H, Silman I, Sussman JL, Weik M: Induced-fit or preexisting equilibrium dynamics? Lessons from protein crystallography and MD simulations on acetylcholinesterase and implications for structure-based drug design. Protein Sci 2008, 17:601-605.

4. Silman I, Sussman JL: Acetylcholinesterase: 'classical' and 'non-classical' functions and pharmacology. Curr Opin Pharmacol 2005, 5:293-302.

5. Inestrosa NC, Alvarez A, Perez CA, Moreno RD, Vicente M, Linker C, Casanueva Ol, Soto C, Garrido J: Acetylcholinesterase accelerates assembly of amyloid- $\beta$-peptides into Alzheimer's fibrils: possible role of the peripheral site of the enzyme. Neuron 1996, 16:881-891.

6. Inestrosa NC, Dinamarca MC, Alvarez A: Amyloid-cholinesterase interactions. Implications for Alzheimer's disease. FEBS J 2008, 275:625-632.
7. Rees T, Hammond Pl, Soreq H, Younkin S, Brimijoin S: Acetylcholinesterase promotes beta-amyloid plaques in cerebral cortex. Neurobiol Aging 2003, 24:777-787.

8. Rees TM, Berson A, Sklan EH, Younkin L, Younkin S, Brimijoin S, Soreq H: Memory deficits correlating with acetylcholinesterase splice shift and amyloid burden in doubly transgenic mice. Curr Alzheimer Res 2005, 2:291-300

9. De Ferrari GV, Canales MA, Shin I, Weiner LM, Silman I, Inestrosa NC: A structural motif of acetylcholinesterase that promotes amyloid $\beta$-peptide fibril formation. Biochemistry 2001, 40:10447-10457.

10. Harel M, Schalk I, Ehret-Sabatier L, Bouet F, Goeldner M, Hirth C, Axelsen PH, Silman I, Sussman JL: Quaternary ligand binding to aromatic residues in the active-site gorge of acetylcholinesterase. Proc Natl Acad Sci USA 1993, 90:9031-9035.

11. Silman I, Harel M, Axelsen P, Raves M, Sussman JL: Three-dimensional structures of acetylcholinesterase and of its complexes with anticholinesterase agents. Biochem Soc Trans 1994, 22:745-749.

12. Harel M, Quinn DM, Nair HK, Silman I, Sussman JL: The X-ray structure of a transition state analog complex reveals the molecular origins of the catalytic power and substrate specificity of acetylcholinesterase. J Am Chem Soc 1996, 118:2340-2346.

13. Greenblatt HM, Kryger G, Lewis T, Silman I, Sussman JL: Structure of acetylcholinesterase complexed with (-)-galanthamine at $2.3 \mathrm{~A}$ resolution. FEBS Lett 1999, 463:321-326.

14. Kryger G, Silman I, Sussman JL: Three-dimensional structure of a complex of E2020 with acetylcholinesterase from Torpedo californica. J Physiol Paris 1998, 92:191-194.

15. Botti SA, Felder CE, Lifson S, Sussman JL, Silman I: A modular treatment of molecular traffic through the active site of cholinesterase. Biophys J 1999, 77:2430-2450.

16. Sussman JL, Harel M, Frolow F, Oefner C, Goldman A, Toker L, Silman I: Atomic structure of acetylcholinesterase from Torpedo californica: a prototypic acetylcholine-binding protein. Science 1991, 253:872-879.

17. Ordentlich A, Barak D, Kronman C, Flashner Y, Leitner M, Segall Y, Ariel N, Cohen S, Velan B, Shafferman A: Dissection of the human acetylcholinesterase active center determinants of substrate specificity. Identification of residues constituting the anionic site, the hydrophobic site, and the acyl pocket. J Biol Chem 1993, 268:17083-17095.

18. Barak D, Kronman C, Ordentlich A, Ariel N, Bromberg A, Marcus D, Lazar A Velan B, Shafferman A: Acetylcholinesterase peripheral anionic site degeneracy conferred by amino acid arrays sharing a common core. $J$ Biol Chem 1994, 269:6296-6305

19. Berman HM, Westbrook J, Feng Z, Gilliland G, Bhat TN, Weissig H, Shindyalov IN, Bourne PE: The Protein Data Bank. Nucleic Acids Res 2000, 28:235-242.

20. DiMasi JA, Hansen RW, Grabowski HG: The price of innovation: new estimates of drug development costs. J Health Econ 2003, 22:151-185.

21. Ertl P: Cheminformatics analysis of organic substituents: identification of the most common substituents, calculation of substituent properties, and automatic identification of drug-like bioisosteric groups. J Chem Inf Comput Sci 2003, 43:374-380.

22. Walters WP, Stahl MT, Murcko MA: Virtual screening-an overview. Drug Discov Today 1998, 3:160-178.

23. Lakshmi PJ, Kumar BV, Nayana RS, Mohan MS, Bolligarla R, Das SK, Bhanu MU, Kondapi AK, Ravikumar M: Design, synthesis, and discovery of novel nonpeptide inhibitor of Caspase-3 using ligand based and structure based virtual screening approach. Bioorg Med Chem 2009, 17:6040-6047.

24. Wei D, Jiang X, Zhou L, Chen J, Chen Z, He C, Yang K, Liu Y, Pei J, Lai L: Discovery of multitarget inhibitors by combining molecular docking with common pharmacophore matching. J Med Chem 2008, 51:7882-7888.

25. Bolognesi ML, Banzi R, Bartolini M, Cavalli A, Tarozzi A, Andrisano V, Minarini A, Rosini M, Tumiatti $V$, Bergamini C: Novel class of quinonebearing polyamines as multi-target-directed ligands to combat Alzheimer's disease. J Med Chem 2007, 50:4882-4897.

26. Bolognesi ML, Cavalli A, Valgimigli L, Bartolini M, Rosini M, Andrisano V, Recanatini M, Melchiorre C: Multi-target-directed drug design strategy: from a dual binding site acetylcholinesterase inhibitor to a trifunctional compound against Alzheimer's disease. J Med Chem 2007, 50:6446-6449.

27. Piazzi L, Belluti F, Bisi A, Gobbi S, Rizzo S, Bartolini M, Andrisano V, Recanatini M, Rampa A: Cholinesterase inhibitors: SAR and enzyme inhibitory activity of 3-[ $\omega$-(benzylmethylamino)alkoxy]xanthen-9-ones. Bioorg Med Chem 2007, 15:575-585. 
28. Piazzi L, Cavalli A, Belluti F, Bisi A, Gobbi S, Rizzo S, Bartolini M, Andrisano V, Recanatini M, Rampa A: Extensive SAR and Computational Studies of 3\{4-[(Benzylmethylamino)methyl]phenyl\}-6,7-dimethoxy-2H- 2chromenone (AP2238) Derivatives. J Med Chem 2007, 50:4250-4254.

29. Camps P, Formosa X, Galdeano C, Go mez T, Mun oz-Torrero D, Scarpellini M, Viayna E, Badia A, Clos MV, Camins A: Novel Donepezil-Based Inhibitors of Acetyl- and Butyrylcholinesterase and AcetylcholinesteraseInduced $\beta$-Amyloid Aggregation. J Med Chem 2008, 51:3588-3598.

30. Camps P, Formosa X, Galdeano C, Munoz-Torrero D, Ramirez L, Gomez E, Isambert N, Lavilla R, Badia A, Clos MV: Pyrano[3,2-c]quinoline-6Chlorotacrine Hybrids as a Novel Family of Acetylcholinesterase- and $\beta$ Amyloid-Directed Anti-Alzheimer Compounds. J Med Chem 2009, 52:5365-5379.

31. Rizzo S, Riviére C, Piazzi L, Bisi A, Gobbi S, Bartolini M, Andrisano V, Morroni F, Tarozzi A, Monti JP: Benzofuran-Based Hybrid Compounds for the Inhibition of Cholinesterase Activity, $\beta$ Amyloid Aggregation, and $A \beta$ Neurotoxicity. J Med Chem 2008, 51:2883-2886.

32. Rosini M, Simoni E, Bartolini M, Cavalli A, Ceccarini L, Pascu N, McClymont DW, Tarozzi A, Bolognesi ML, Minarini A: Inhibition of Acetylcholinesterase, $\beta$-Amyloid Aggregation, and NMDA Receptors in Alzheimer's Disease: A Promising Direction for the Multi-target-Directed Ligands Gold Rush. J Med Chem 2008, 51:4381-4384.

33. Tumiatti $\vee$, Milelli A, Minarini A, Rosini M, Bolognesi ML, Micco M, Andrisano V, Bartolini M, Mancini F, Recanatini M: Structure-Activity Relationships of Acetylcholinesterase Noncovalent Inhibitors Based on a Polyamine Backbone. 4. Further Investigation on the Inner Spacer. J Med Chem 2008, 51:7308-7312.

34. Brooks BR, Bruccoleri RE, Olafson BD: CHARMM: A program for macromolecular energy, minimization, and dynamics calculations. J Comput Chem 1983, 4:187-217.

35. Li H, Sutter J, Hoffmann RD: HypoGen: An automated system for generating 3D predictive pharmacophore models. In Pharmacophore perception, development, and use in drug design, IUL Biotechnology Series. Edited by: Güner OF. La Jolla, CA: International University Line; 2000:171-189.

36. Sutter J, Güner O, Hoffmann RD, Li H, Waldman M: Effect of Variable Weights and Tolerances on Predictive Model Generation. In Pharmacophore perception, development, and use in drug design, IUL Biotechnology Series. Edited by: Güner OF. La Jolla, CA: International University Line; 2000:501-511.

37. Kurogi Y, Güner OF: Pharmacophore modeling and three-dimensional database searching for drug design using catalyst. Curr Med Chem 2001, 8:1035-1055

38. Poptodorov K, LuU T, Hoffmann RD: In Methods and principles in Medicinal Chemistry, Pharmacophores and Pharmacophores Searches. Edited by: Langer T, Hoffmann RD. Germany: Wiley-VCH:Weinheim; 2006:2:17-47.

39. Liu S, Neidhardt EA, Grossman TH, Ocain T, Clardy J: Structures of human dihydroorotate dehydrogenase in complex with antiproliferative agents. Structure 2000, 8:25-33.

40. Güner OF, Henry DR: Metric for analyzing hit lists and pharmacophores. In Pharmacophore perception, development, and use in drug design, IUL Biotechnology Series. Edited by: Güner OF. La Jolla, CA: International University Line; 2000:191-212.

41. Güner OF, Waldman M, Hoffmann RD, Kim JH: Strategies for database mining and pharmacophore development, 1st. In Pharmacophore perception, development, and use in drug design, IUL Biotechnology Series. Edited by: Güner OF. La Jolla: International University Line; 2000:213-236.

42. Clement OO, Freeman CM, Hartmann RW, Handratta VD, Vasaitis TS, Brodie AM, Njar VC: Three dimensional pharmacophore modeling of human CYP17 inhibitors. Potential agents for prostate cancer therapy. J Med Chem 2003, 46:2345-2351.

43. Butini S, Campiani G, Borriello M, Gemma S, Panico A, Persico M, Catalanotti B, Ros S, Brindisi M, Agnusdei M, et al: Exploiting protein fluctuations at the active-site gorge of human cholinesterases: further optimization of the design strategy to develop extremely potent inhibitors. J Med Chem 2008, 51:3154-3170.

44. Campiani G, Fattorusso C, Butini S, Gaeta A, Agnusdei M, Gemma S, Persico M, Catalanotti B, Savini L, Nacci V, Novellino E, Holloway HW, Greig NH, Belinskaya T, Fedorko JM, Saxena A: Development of molecular probes for the identification of extra interaction sites in the mid-gorge and peripheral sites of butyrylcholinesterase (BuChE). Rational design of novel, selective, and highly potent BuChE inhibitors. J Med Chem 2005, 48:1919-1929.

45. Cavalli A, Bolognesi ML, Minarini A, Rosini M, Tumiatti V, Recanatini M, Melchiorre C: Multi-target-directed ligands to combat neurodegenerative diseases. J Med Chem 2008, 51:347-372.

46. Rodriguez-Franco MI, Fernandez-Bachiller MI, Perez C, HernandezLedesma B, Bartolome B: Novel tacrine-melatonin hybrids as dual-acting drugs for Alzheimer disease, with improved acetylcholinesterase inhibitory and antioxidant properties. J Med Chem 2006, 49:459-462.

47. Huang N, Shoichet BK, Irwin JJ: Benchmarking sets for molecular docking. J Med Chem 2006, 49:6789-6801.

48. Muegge I: Selection criteria for drug-like compounds. Med Res Rev 2003, 23:302-321.

49. Lipinski CA, Lombardo F, Dominy BW, Feeney PJ: Experimental and computational approaches to estimate solubility and permeability in drug discovery and development settings. Adv Drug Deliv Rev 2001, 46:3-26.

50. Kryger G, Harel M, Giles K, Toker L, Velan B, Lazar A, Kronman C, Barak D, Ariel N, Shafferman A, Silman I, Sussman JL: Structures of recombinant native and E202Q mutant human acetylcholinesterase complexed with the snake-venom toxin fasciculin-II. Acta Crystallogr Sect D-Biol Crystallogr 2000, 56:1385-1394.

51. Diller DJ, Merz KM: High throughput docking for library design and library prioritization. Proteins: Struct Funct Bioinf 2001, 43:113-124.

52. Marcu MG, Chadli A, Bouhouche I, Catelli M, Neckers LM: The heat shock protein 90 antagonist novobiocin interacts with a previously unrecognized ATP-binding domain in the carboxyl terminus of the chaperone. J Biol Chem 2000, 275:37181-37186.

53. Shen T, Tai K, Henchman RH, McCammon JA: Molecular dynamics of acetylcholinesterase. Accounts Chem Res 2002, 35:332-340.

doi:10.1186/1423-0127-18-8

Cite this article as: Lu et al:: The discovery of potential acetylcholinesterase inhibitors: A combination of pharmacophore modeling, virtual screening, and molecular docking studies. Journal of Biomedical Science 2011 18:8.

\section{Submit your next manuscript to BioMed Central and take full advantage of:}

- Convenient online submission

- Thorough peer review

- No space constraints or color figure charges

- Immediate publication on acceptance

- Inclusion in PubMed, CAS, Scopus and Google Scholar

- Research which is freely available for redistribution

Submit your manuscript at www.biomedcentral.com/submit
C Biomed Central 